\title{
Temperature and aridity regulate spatial variability of soil multifunctionality in drylands across the globe
}

\author{
Jorge Durán (iD,${ }^{1,9}$ Manuel Delgado-Baquerizo, ${ }^{2,3}$ Andrew J. Dougill ${ }^{4}$ Reginald T. Guuroh, ${ }^{5,6}$ \\ Anja Linstädter (iD ${ }^{6,7}$ Andrew D. Thomas, ${ }^{8}$ and Fernando T. Maestre ${ }^{3}$ \\ ${ }^{1}$ Centre for Functional Ecology, University of Coimbra, 3000-456 Coimbra, Portugal \\ ${ }^{2}$ Cooperative Institute for Research in Environmental Sciences, University of Colorado, Boulder, Colorado 80309, USA \\ ${ }^{3}$ Departamento de Biología y Geología, Física y Química Inorgánica, Escuela Superior de Ciencias Experimentales y Tecnología, Universidad \\ Rey Juan Carlos, Móstoles 28933, Spain \\ ${ }^{4}$ School of Earth and Environment, University of Leeds, Leeds LS2 9JT, United Kingdom \\ ${ }^{5}$ CSIR-Forestry Research Institute of Ghana, KNUST, Kumasi P.O. Box UP 63, Ghana \\ ${ }^{6}$ Range Ecology and Range Management Group, Botanical Institute, University of Cologne, Zuelpicher Strasse 47b, 50674 Cologne, Germany \\ ${ }^{7}$ INRES - Institute of Crop Science and Resource Conservation, University of Bonn, Karlrobert-Kreiten-Str. 13, 53113 Bonn, Germany \\ ${ }^{8}$ Department of Geography and Earth Sciences, Aberystwyth University, Aberystwyth SY23 3DB, United Kingdom
}

\begin{abstract}
The relationship between the spatial variability of soil multifunctionality (i.e., the capacity of soils to conduct multiple functions; SVM) and major climatic drivers, such as temperature and aridity, has never been assessed globally in terrestrial ecosystems. We surveyed 236 dryland ecosystems from six continents to evaluate the relative importance of aridity and mean annual temperature, and of other abiotic (e.g., texture) and biotic (e.g., plant cover) variables as drivers of SVM, calculated as the averaged coefficient of variation for multiple soil variables linked to nutrient stocks and cycling. We found that increases in temperature and aridity were globally correlated to increases in SVM. Some of these climatic effects on SVM were direct, but others were indirectly driven through reductions in the number of vegetation patches and increases in soil sand content. The predictive capacity of our structural equation modelling was clearly higher for the spatial variability of $\mathrm{N}$ - than for $\mathrm{C}$ - and P-related soil variables. In the case of $\mathrm{N}$ cycling, the effects of temperature and aridity were both direct and indirect via changes in soil properties. For $\mathrm{C}$ and $\mathrm{P}$, the effect of climate was mainly indirect via changes in plant attributes. These results suggest that future changes in climate may decouple the spatial availability of these elements for plants and microbes in dryland soils. Our findings significantly advance our understanding of the patterns and mechanisms driving SVM in drylands across the globe, which is critical for predicting changes in ecosystem functioning in response to climate change.
\end{abstract}

Key words: carbon cycling; climate change; multifunctionality; nitrogen cycling; phosphorous cycling; spatial heterogeneity.

\section{INTRODUCTION}

The uneven distribution of soil characteristics is a ubiquitous feature of most terrestrial ecosystems, and is known to regulate a wide range of ecosystem processes including individual plant performance and competitive ability (Day et al. 2003), community-level productivity (Maestre and Reynolds 2006), trophic interactions (Tsunoda et al. 2014), and nutrient cycling (Schlesinger et al. 1990, Ochoa-Hueso et al. 2018). This spatial variability in soil variables (SV hereafter) is largely controlled by the interaction of multiple biological, chemical, and physical processes acting simultaneously at multiple scales (Jackson and Caldwell 1993, Farley and Fitter 1999, Zuo et al. 2010). While the environmental drivers of SV have been studied on individual functions and at local scales (e.g., $\mathrm{N}$ availability), the role of environment in driving multiple ecosystem functions simultaneously still needs to be considered to achieve an integrative understanding on the drivers of within-site SV (Byrnes et al. 2014).

Given the ubiquitous nature of SV and its role as a modulator of plant and soil fauna responses to climate change

Manuscript received 3 November 2017; revised 16 January 2018; accepted 30 January 2018. Corresponding Editor: Scott L. Collins.

${ }^{9}$ E-mail: jdh@uc.pt
(García-Palacios et al. 2012), a deeper understanding of the major drivers of the spatial variability in multiple soil functions (i.e., soil multifunctionality, SVM) across the globe is of paramount importance to anticipate changes in ecosystem functioning under global change scenarios (Fraterrigo and Rusak 2008, IPCC 2013). Remarkably, a relatively large body of literature has explored the effects of SV locally or in regional comparisons (Linstädter et al. 2014, Guuroh et al. 2018), but no study has yet assessed the major environmental drivers of SVM in terrestrial ecosystems across the globe, which remains largely unexplored and poorly understood. This is particularly relevant for dryland ecosystems (i.e., arid, semiarid, and dry-subhumid ecosystems), where SVM is a widespread phenomenon (Schlesinger et al. 1990, Ochoa-Hueso et al. 2018). In drylands, which typically have a patchy plant distribution, SVM likely arises from the strong functional differences between vegetated patches, where plants largely drive biological processes such as litter decomposition or $\mathrm{N}$ fixation, and unvegetated areas, with higher importance of physical processes such as erosion by wind or water (Li et al. 2007). Drylands cover about $45 \%$ of Earth's land surface and support more than $38 \%$ of the global human population (Prăvălie 2016), and their global extent may increase by up to $23 \%$ by the end of this century due to forecasted increases in aridity (Huang et al. 2016). 
These areas are particularly sensitive to the effects of climate change, so expected increases in aridity and temperature (up to $2-4^{\circ} \mathrm{C}$, depending on projections; IPCC 2013 ) will promote changes in vegetation and soil properties that could have significant consequences for SVM in drylands worldwide. For instance, recent field surveys have found that increases in aridity are linked to decreases in plant cover and to increases in the encroachment rate of woody vegetation across the globe (Dougill and Thomas 2004, Vicente-Serrano et al. 2012, Delgado-Baquerizo et al. 2013), phenomena that would likely lead to parallel increases in SVM. However, to date, no study has considered the multiple direct and indirect (e.g., via plant and soil features) effects of temperature or aridity on SVM. The likely influence of climate on SVM, together with the well-known influence of SVM on ecosystem functioning, anticipate changes in the ability of dryland ecosystems to provide goods and services under ongoing climate change. This, together with the global importance of drylands and their particular sensitivity to climatic changes, justify efforts to better understand the role of major climate change drivers, such as temperature and aridity, in determining SVM globally directly and indirectly via plant and soil attributes.

Here, we used a database including 236 dryland sites from six continents (Appendix S1: Fig. S1) to evaluate the role and relative importance of aridity and mean annual temperature, together with other key environmental factors (soil properties and plant attributes), as drivers of SVM in drylands worldwide. We hypothesized that increases in aridity and temperature will promote SVM directly, but also indirectly via reductions in plant cover and shifts in soil properties, as plant community composition and structure are largely known to be major drivers of soil spatial variability in drylands (Schlesinger et al. 1990).

\section{Methods}

We used data from a global field survey conducted in 236 dryland sites from 19 countries (Appendix S1: Fig. S1; Argentina, Australia, Botswana, Brazil, Burkina Faso, Chile, China, Ecuador, Ghana, Iran, Israel, Kenya, Mexico, Morocco, Peru, Spain, Tunisia, USA, and Venezuela). These sites include the 224 drylands used in Maestre et al. (2012) plus 12 additional sites from Botswana, Ghana, and Burkina Faso surveyed in 2012 and 2013. All sites surveyed were restricted to dryland ecosystems (defined as regions with an aridity index $[\mathrm{AI}=$ mean precipitation/mean potential evapotranspiration] between 0.05 and 0.65 [Gao and Giorgi 2008]) and encompassed a wide variety of dryland vegetation types, including grasslands, shrublands, savannas, dry seasonal forests, and open, tree-dominated woodlands. Mean annual precipitation and temperature of the study sites ranged from 66 to $1219 \mathrm{~mm}$, and from $-1.8^{\circ}$ to $27.8^{\circ} \mathrm{C}$.

Data collection was carried out between February 2006 and December 2013, focusing on $30 \times 30 \mathrm{~m}$ plots representative of the vegetation at each site and using a standardized sampling protocol (Maestre et al. 2012). Soils were sampled during the dry season in most of the sites using a stratified random procedure. At each plot, five $50 \times 50 \mathrm{~cm}$ quadrats were randomly placed under the canopy of the dominant perennial species and in open areas devoid of perennial vegetation. A composite sample consisting of five $145-\mathrm{cm}^{3}$ soil cores $(0-7.5 \mathrm{~cm}$ depth) was collected from each quadrat, bulked, and homogenized in the field. When more than one dominant plant species was present, samples were also collected under the canopies of five randomly selected individuals of the co-dominant species. Thus, the number of soil samples varied between 10 and 15 per site. Soil samples were taken to the laboratory, sieved ( $2 \mathrm{~mm}$ mesh), air-dried for one month, and stored for laboratory analyses. In drylands, soil properties remain largely similar after air drying (the most common status for these soils), so we did not expect large changes in soil properties after air drying (Zornoza et al. 2006, 2009). To facilitate the comparison of results across sites, dried soil samples from all sites were shipped to Spain for laboratory analyses.

We measured the cover and number of perennial plant patches at each site using the line-intercept method along four $30 \mathrm{~m}$ long transects separated from each other by $8 \mathrm{~m}$ (Brun and Box 2006). At each transect, we also surveyed 20 contiguous $1.5 \times 1.5 \mathrm{~m}$ quadrats ( 80 quadrats per site). Within these quadrats, we counted the number of species present to estimate species richness. Soil $\mathrm{pH}$ was measured with a $\mathrm{pH}$ meter, in a 1:2.5 (mass: volume, soil:water) suspension. Soil sand content was estimated according to (Kettler et al. 2001). Mean annual temperature (MAT) and aridity ( 1 - aridity index [ratio of precipitation to potential evapotranspiration]) were obtained from Zomer et al. (2008), who used interpolations from the Worldclim global database (Hijmans et al. 2005).

We measured 14 variables closely related to $\mathrm{C}, \mathrm{N}$, and $\mathrm{P}$ cycling and storage. Total $\mathrm{N}$ was determined using a $\mathrm{CN}$ analyzer (Leco CHN628 Series; Leco Corporation, St. Joseph, Michigan, USA). Total organic $\mathrm{C}$ was determined by colorimetry after oxidation with a mixture of potassium dichromate and sulfuric acid (Anderson and Ingram 1993). Soil pentoses and hexoses, as well as soil ammonium and nitrate were measured colorimetrically from soil extracts as described in Delgado-Baquerizo et al. (2015). Soil samples $(2.5 \mathrm{~g}$ of soil) were extracted with $0.5 \mathrm{~mol} / \mathrm{L}$ $\mathrm{K}_{2} \mathrm{SO}_{4}$ in a ratio of $1: 5$. Extracts were shaken in an orbital shaker at $200 \mathrm{rpm}$ for $1 \mathrm{~h}$ at $20^{\circ} \mathrm{C}$ and filtered to pass a $0.45-\mu \mathrm{m}$ Millipore filter (Jones and Willett 2006). Potential net $\mathrm{N}$ mineralization rate was estimated as the difference between initial and final inorganic $\mathrm{N}$ (sum of ammonium and nitrate) before and after incubation under potential conditions (Allen et al. 1986). Phosphatase activity was measured by determination of the amount of pnitrophenol released from $0.5 \mathrm{~g}$ soil after incubation at $37^{\circ} \mathrm{C}$ for $1 \mathrm{~h}$ with the substrate p-nitrophenyl phosphate in MUB buffer (Tabatabai and Bremner 1969). The activity of b-glucosidase was assayed following the procedure for phosphatase, but using p-nitrophenyl-b-D-glucopyranoside as substrate and trishydroxymethyl aminomethane instead of $\mathrm{NaOH}$ when preparing the buffer (Tabatabai 1982). Available phosphorus was determined colorimetrically from sodium bicarbonate extracts (Moir and Tiessen 2007). Soil proteins, phenols and aromatic compounds were evaluated as detailed in Maestre et al. (2012). These variables were selected because they are considered good proxies of key ecosystem processes linked to soil fertility, 
nutrient cycling, biological productivity, and the build-up of nutrient pools.

To estimate SVM, we first calculated the variability within each site of the different soil variables measured. To do so, we calculated the site-level coefficient of variation (CV) using the composite soil samples obtained at each site ( $n$ per site varied between 10 and 15; see above). The CV is a relative measure of heterogeneity that can accommodate variance-mean scaling, avoiding the tendency for variance to increase with the mean (Duarte 1991). Therefore, it has been shown to be more useful than absolute measures of variability such as the standard deviation for comparing variability within biological properties (Schlesinger et al. 1990, Fraterrigo and Rusak 2008). We calculated SVM as the arithmetic mean for all individual site-level CVs of all soil variables. We also calculated the SVM of C-, N-, and P-related variables separately (hereafter C-SVM, N-SVM, and P-SVM) by calculating the arithmetic mean of individual site-level CVs of soil variables related to the $\mathrm{C}$ (i.e., total organic $\mathrm{C}$, activity of b-glucosidase, phenols, aromatic compounds, hexoses, and pentoses), N (total nitrogen, ammonium, nitrate, proteins, potential nitrification rate, and amino acids), and $\mathrm{P}$ (available phosphorus, and phosphatase activity) cycles.

To evaluate the effects of biotic and abiotic factors on SVM, we first explored the relationships between MAT, aridity, and SVM using regression analysis. Then we used random forest modelling (Breiman 2001) to identify the most important predictors of SVM among the following: latitude, longitude, mean annual temperature, aridity, plant species richness, plant cover, number of plant patches, the ratio between woody and herbaceous cover, soil sand, and soil $\mathrm{pH}$. Finally, to achieve a system-level understanding of the major drivers of SVM, we used structural equation modelling (SEM; Grace 2006). In particular, we used SEM to evaluate the multiple direct and indirect effects of biotic and abiotic drivers on SVM (our a priori model based on our current knowledge is available in Appendix S1: Fig. S2). Structural equation modelling is particularly useful in large-scale correlative studies because it allows us to partition causal influences among multiple variables and to separate the direct and indirect effects of the predictors included in the model (Grace 2006). Variables were log- or square-root-transformed when necessary to improve linearity in the relationships in our SEM models. There is no single universally accepted test of overall goodness of fit for SEM, applicable in all situations regardless of sample size or data distribution. Here we used the chi-squared test $\left(\chi^{2}\right.$; the model has a good fit when $\chi^{2}$ is low, i.e., $\sim \leq 2$, and $P$ is high, traditionally $>0.05$ ) and the root-mean-square error of approximation (RMSEA; the model has a good fit when RMSEA is low, i.e., $\sim 0.05$, and $P$ is high, traditionally $>0.05$; Schermelleh-Engel et al. 2003). After verifying the adequate fit of our model, we were free to interpret the path coefficients of the model and their associated $P$ values. A path coefficient is analogous to the partial correlation coefficient, and describes the strength and sign of the relationships between two variables (Grace 2006). The probability that a path coefficient differs from zero was tested using bootstrap tests (SchermellehEngel et al. 2003). We calculated the standardized total effects of all biotic and abiotic drivers on the selected heterogeneity metrics (Grace 2006). The net influence that one variable had upon another was calculated by summing all direct and indirect pathways (effects) between two variables. Then we parameterized the model using our data set and tested its overall goodness of fit. All SEM and regression analyses were conducted using AMOS 20 (IBM SPSS, Chicago, Illinois, USA) and Sigma Plot 12, respectively (Systat Software, San José, California, USA). Random forest modelling was performed with R 3.3.2 using the rfPermute package (R Core Team, Vienna, Austria).

\section{RESULTS}

Regression analyses showed a positive quadratic relationship between SVM and mean annual temperature (MAT; Fig. 1a) and aridity (Fig. 1b). The random forest models indicated that all explored environmental factors were significant predictors of SVM, N-SVM, C-SVM, and P-SVM, except plant cover and plant richness in the case of P-SVM (Fig. 2). These models identified, in this order, (1) soil sand content, MAT, the number of plant patches per plot, soil $\mathrm{pH}$, and aridity as the major individual predictors of SVM (Fig. 2a); (2) MAT, soil $\mathrm{pH}$ and sand content, and aridity for N-SVM (Fig. 2b); (3) soil sand content, the number of plant patches, MAT, and aridity for C-SVM (Fig. 2c); and (4) the number of plant patches, MAT, soil sand content, and aridity for P-SVM (Fig. 2d).

Our SEM explained around $22 \%$ of the variation in SVM (Fig. 3a). We found direct effects from both MAT and aridity on SVM (Fig. 3a). Moreover, we found multiple indirect effects of MAT and aridity on SVM via reductions in number of plant patches (Fig. 3a). Aridity also promoted SVM indirectly via positive effects on sand content. The standardized total effects (sum of direct and indirect effects from SEM) indicated that MAT and aridity were important predictors of SVM, with strong positive effects on SVM in both cases (Fig. 3b). Sand content (positive) and the number of plant patches and soil $\mathrm{pH}$ (negative) were also important drivers of SVM.

The element-specific SEMs explained 26\%, 14\%, and $12 \%$ of the variation observed in $\mathrm{N}-, \mathrm{C}-$, and P-SVM, respectively (Fig. 4). The models showed that N-SVM was positively and significantly related to MAT and soil sand content, but negatively related to soil pH (Fig. 4a), whereas C-SVM and P-SVM were only negatively influenced by the number of plant patches (Figs. 4c,e). The standardized total effects indicated that N-SVM was positively influenced by MAT and soil sand content, but negatively by soil pH (Fig. 4b); C-SVM was positively regulated by aridity and plant cover, but negatively by the number of plant patches and soil pH (Fig. 4d); and PSVM was mainly positively related to aridity and MAT, but strongly and negatively related to the number of plant patches (Fig. 4f).

\section{Discussion}

Our work represents the first global assessment of the major environmental predictors of the spatial variability of multiple surrogates of ecosystem functions (SVM). 

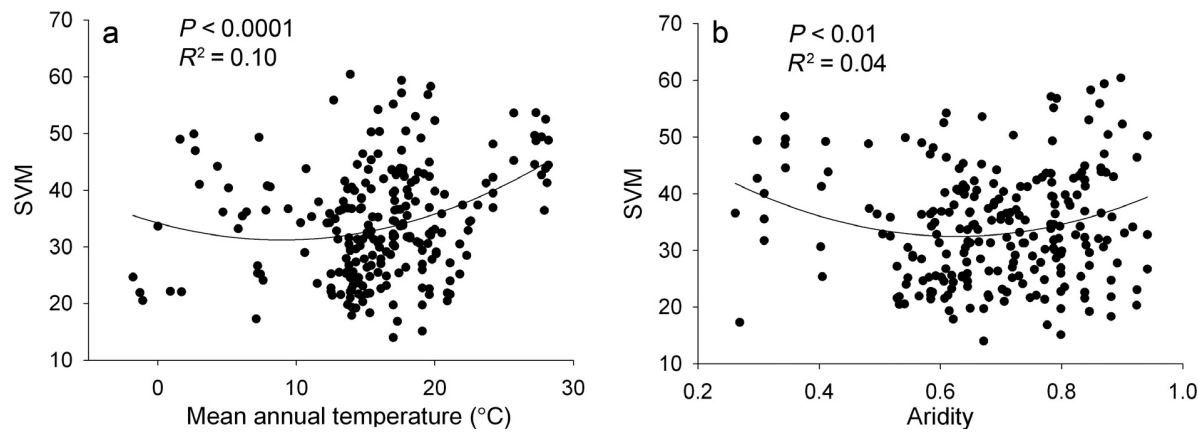

FIG. 1. Relationships between mean annual temperature and aridity (defined as 1 - aridity index [the ratio of precipitation to potential evapotranspiration]) and the variability (coefficient of variation) of soil multifunctionality (SVM). The solid lines represent the fitted regressions. $R^{2}$ shows the proportion of variance explained.
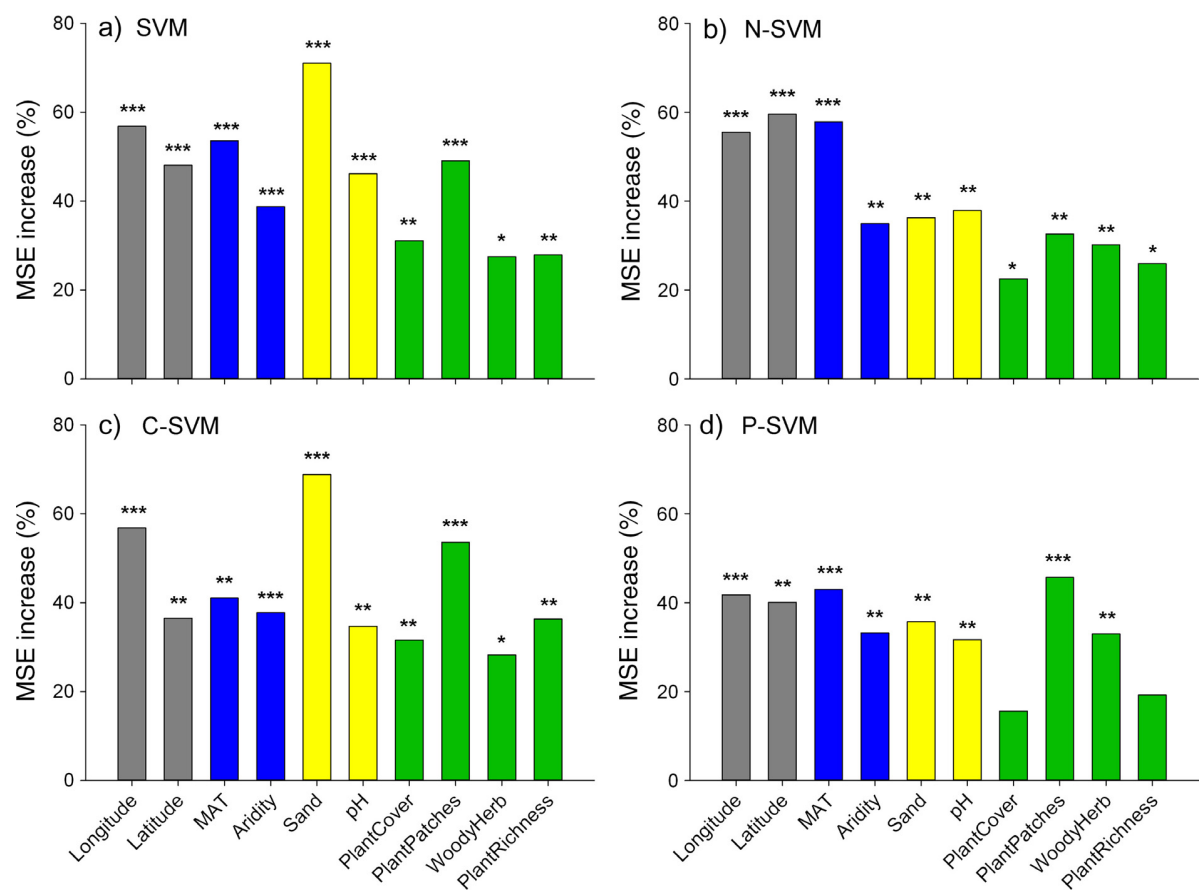

FIG. 2. Random forest mean predictor importance (increase of mean square error [MSE; \%]) of biotic and abiotic drivers on the spatial variability (coefficient of variation) of (a) soil multifunctionality (SVM), (b) N-related variables (SVM-N), (c) C-related variables (SVM-C), and (d) P-related variables (P-SVM). Predictors are latitude, longitude, mean annual temperature (MAT), aridity, plant species richness (PlantRichness), plant cover (PlantCover), number of plant patches (PlantPatches), the ratio between woody and herbaceous cover (WoodyHerb), soil sand (Sand), and soil pH. Significance levels of each predictor are as follows: $* P<0.05, * * P<0.01$, and $* * * P<0.001$.

More importantly, we provide new mechanistic insights indicating that increases in aridity and mean annual temperature are linked to overall increases in the SVM of drylands across the globe both directly and indirectly (via reductions in plant patches and increases in sand content). This study builds on the seminal article from Schlesinger et al. (1990) that illustrated how the loss of vegetation cover due to human-driven disturbances can lead to changes in the heterogeneity of soil resources at the local scale. Although the amount of variance explained by our models is relatively low $\left(R^{2}<0.25\right)$, this is a common outcome in global surveys in which the variability of sampled sites is inevitably high (Moles et al. 2009). Furthermore, it is inherently challenging to characterize the heterogeneity of soil resources, as it can be affected simultaneously by different sources of variability that can operate and affect SVM at different temporal and spatial scales (Fraterrigo and Rusak 2008).

Our results demonstrate that SVM is not only directly related to changes in temperature and aridity, but also emerges from the influence of these climatic variables on vegetation and/or soil features. For instance, increases in aridity enhanced SVM mainly through increases in soil sand content and through reductions in the number of plant patches. On the other hand, increases in MAT were negatively related to SVM through decreases in soil sand 


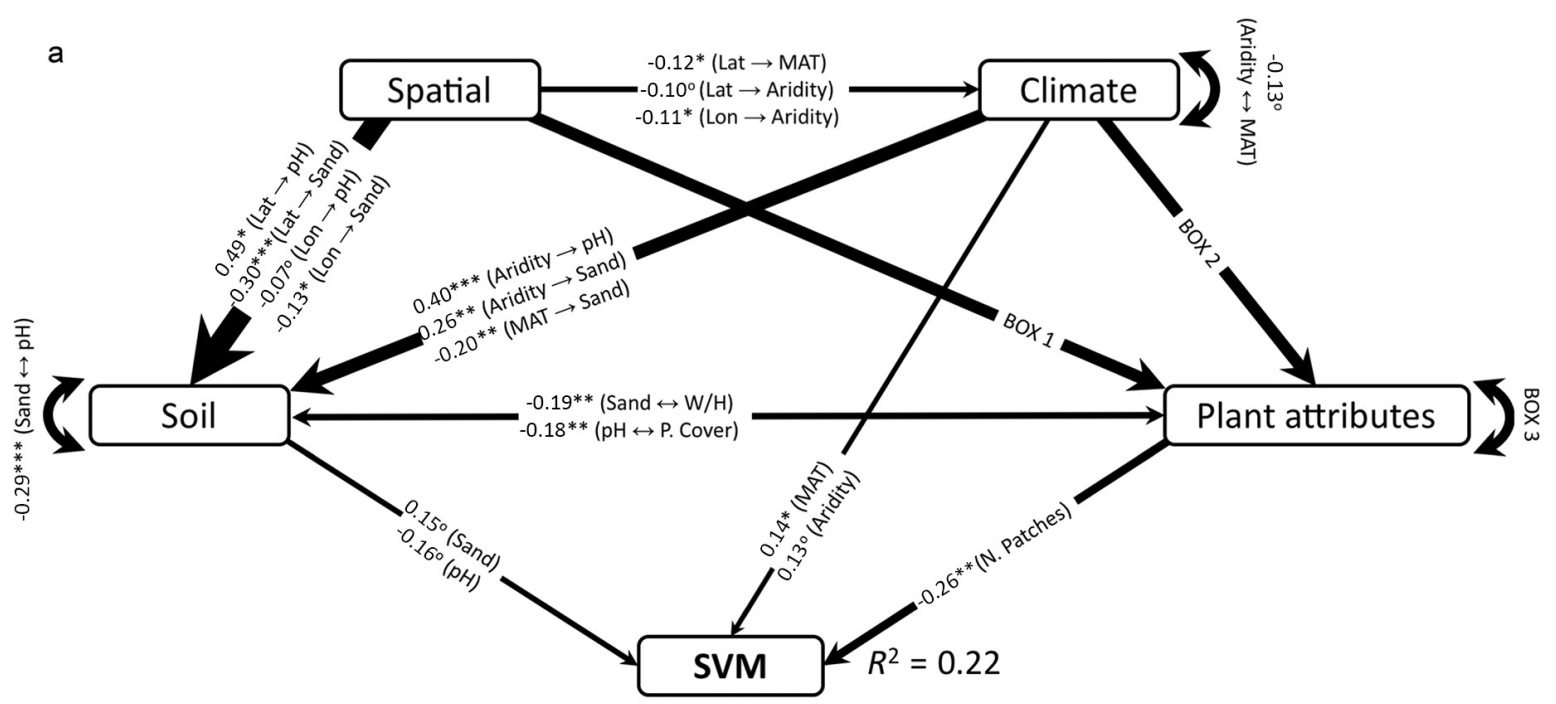

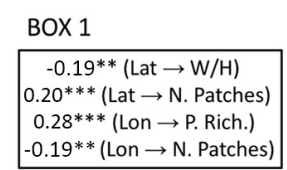

BOX 2

$-0.27^{* *}$ (Aridty $\rightarrow$ P. Rich.)
$0.16^{*}$ (Aridity $\rightarrow$ W/H)
$-0.35^{* *}$ (Aridity $\rightarrow$ N. Patches)
$-0.28^{* *}$ (Aridity $\rightarrow$ P. Cover)
$-0.27^{*}$ (MAT $\rightarrow$ W/H)
$0.16^{*}$ (MAT $\rightarrow$ P. Cover)
$-0.35^{* *}$ (MAT $\rightarrow$ N. Patches)

BOX 3

\begin{tabular}{|c|}
\hline $0.18^{*}$ (P. Cover $\leftrightarrow$ P. Rich.) \\
$-0.43^{* * *}$ (N. Patches $\leftrightarrow$ W/H) \\
$0.18^{* *}$ (P. Cover $\leftrightarrow$ N. Patches) \\
$0.17^{* *}$ (P. Cover $\leftrightarrow$ W/H) \\
$0.15^{*}$ (P. Rich. $\leftrightarrow$ N. Patches) \\
\hline
\end{tabular}

Model fit

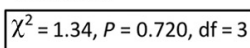

Bootstrap $P=0.738$

RMSEA $=0.00, P=0.857$

b

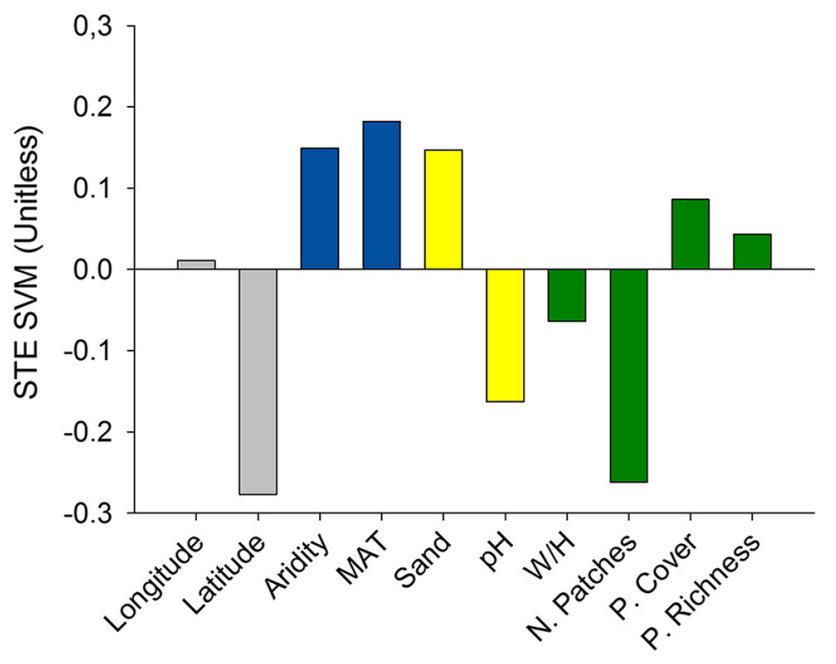

FIG. 3. (a) Effects of mean annual temperature, aridity, soil variables ( $\mathrm{pH}$ and sand content), biotic attributes (plant cover [P. Cover] and richness [P. Rich.], number of plant patches [N. Patches], and woody to herbaceous cover ratio [W/H]), and geographical coordinates (longitude [Lon] and latitude [Lat]) on the spatial variability (coefficient of variation) of soil multifunctionality (SVM). Numbers adjacent to arrows are standardized path coefficients, analogous to relative regression weights, and indicative of the effect size of the relationship. $* P<0.05$ and $* * P<0.01$. Only significant relationships $(P<0.05)$ are shown. Arrow widths are proportional to the strength of the relationship. Squares are observable variables. The proportion of variance explained $\left(R^{2}\right)$ appears alongside the response variable in the model. Goodness-of-fit statistics for each model are shown in the bottom (df, degrees of freedom; RMSEA, root mean squared error of approximation). The components within spatial geolocation, climate, soil (properties) and plant attributes are included as independent observable variables in the model, however, we group them in the same box in the model for graphical simplicity. (b) Standardized total effects (direct plus indirect effects derived from the structural equation models) of SVM predictors.

content. However, this effect was clearly overcome by both the negative effect of MAT on the number of plant patches (which further decreases SVM) and the direct effects of temperature on SVM, resulting in a net positive and strong effect of increasing temperature on SVM.
Results from previous studies conducted at the local scale suggest that any disturbance leading to decreases in vegetation cover (e.g., overgrazing) and increases in woody plant encroachment should increase SV (Schlesinger et al. 1990, Dougill and Thomas 2004) through the development 


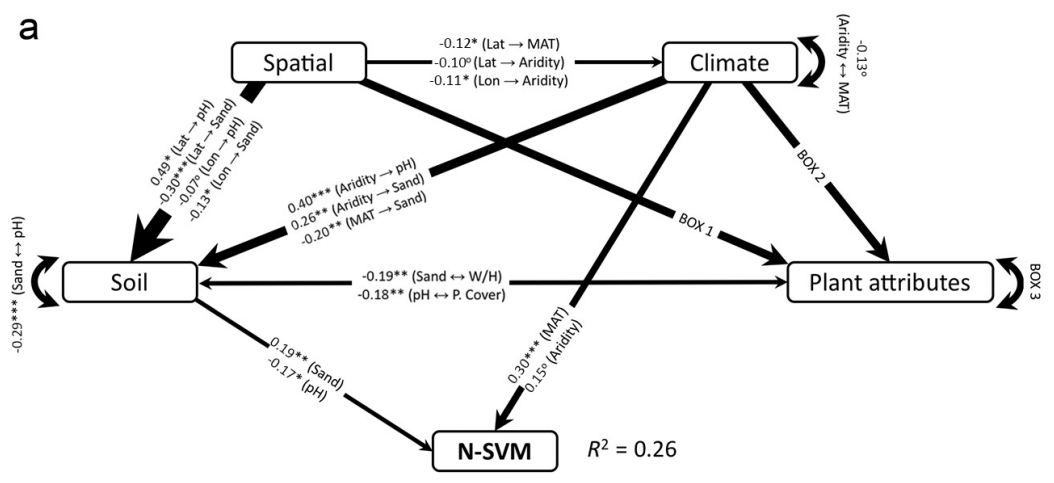

b
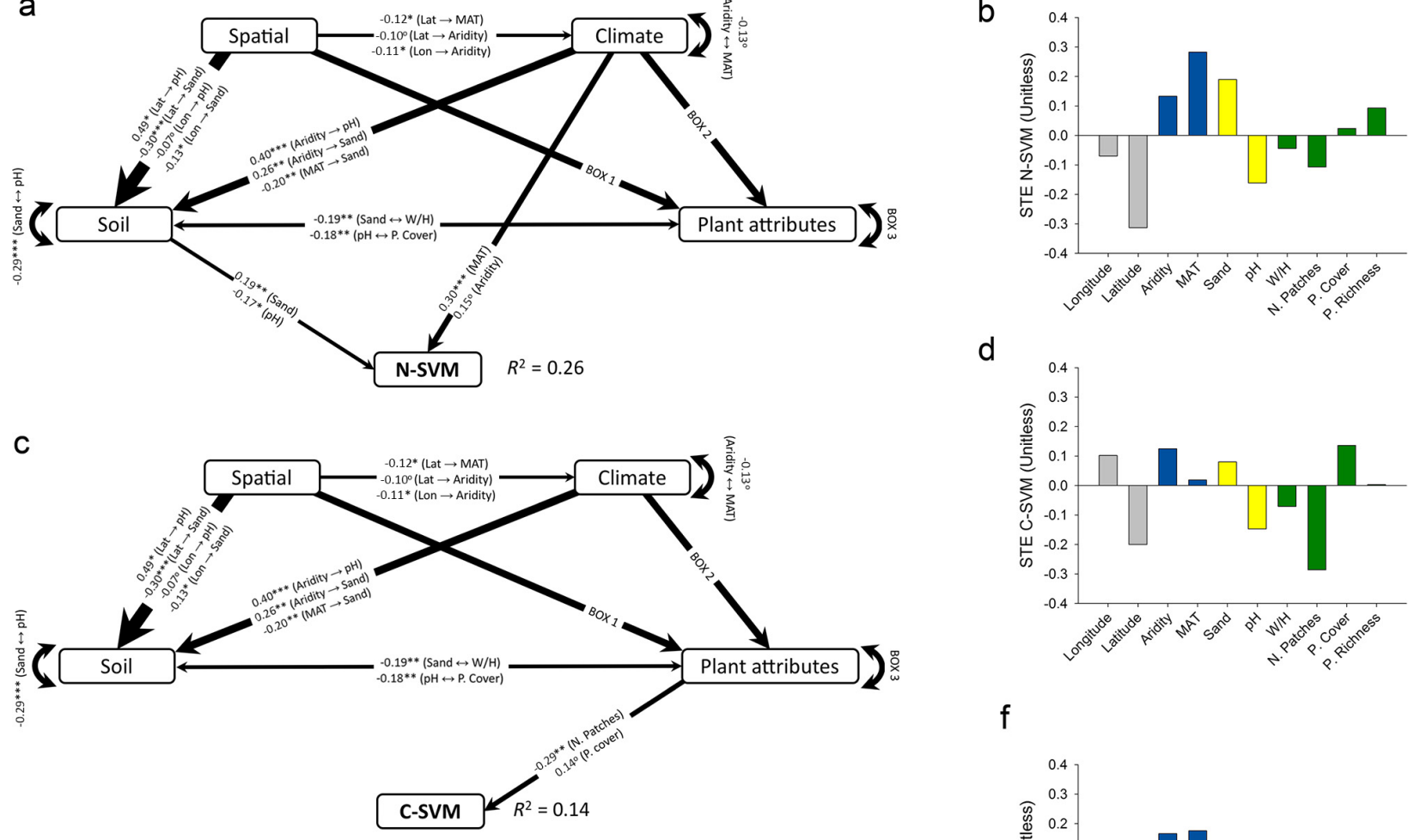

d

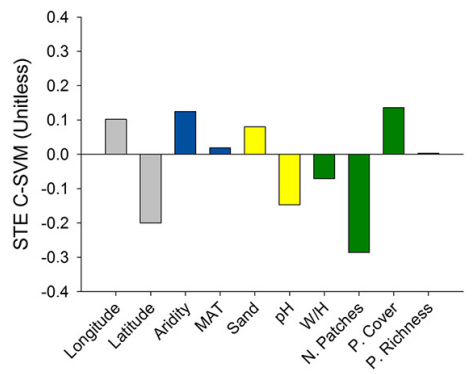

f
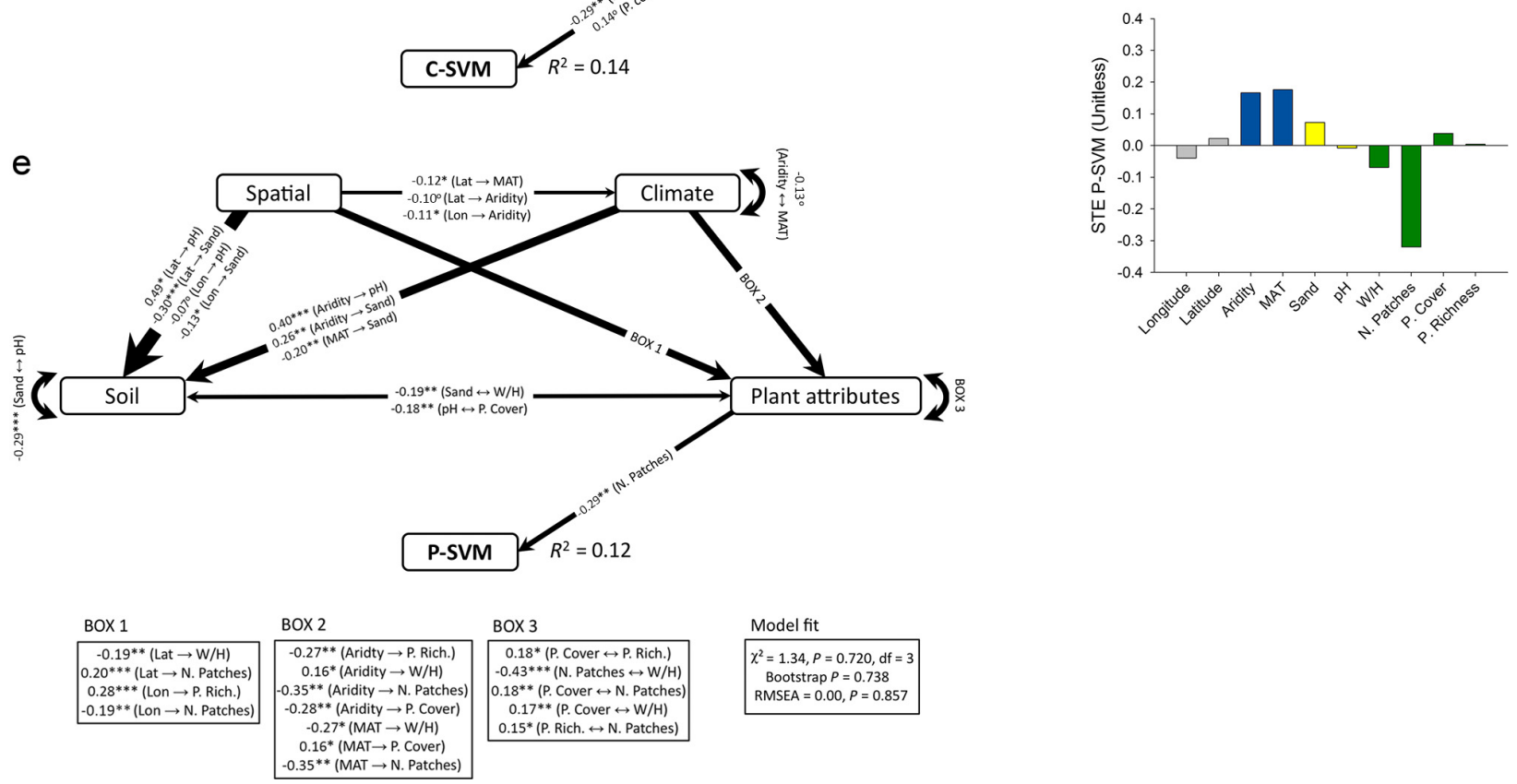

FIG. 4. Effects of mean annual temperature, aridity, soil variables ( $\mathrm{pH}$ and sand content), biotic attributes (plant cover and richness, number of plant patches, and woody to herbaceous cover ratio $[\mathrm{W} / \mathrm{H}]$ ) and geographical coordinates (longitude and latitude) on the spatial variability (coefficient of variation) of the soil multifunctionality of (a) N-, (c) C-, and (e) P-related variables (N-SVM, C-SVM, and PSVM, respectively). Numbers adjacent to arrows are standardized path coefficients, analogous to relative regression weights, and indicative of the effect size of the relationship. $* P<0.05$ and $* * P<0.01$. Only significant relationships $(P<0.05)$ are shown. Arrow widths are proportional to the strength of the relationship. Squares are observable variables. The proportion of variance explained $\left(R^{2}\right)$ appears alongside the response variable in the model. Goodness-of-fit statistics for each model are shown in the bottom $(\mathrm{df}=\mathrm{degrees}$ of freedom; RMSEA = root mean squared error of approximation). The components within spatial geolocation, climate, soil (properties) and plant attributes are included as independent observable variables in the model, however we group them in the same box in the model for graphical simplicity. Panels b, d, and f show the standardized total effects (direct plus indirect effects derived from the structural equation models) of N-SVM, C-SVM, and P-SVM predictors, respectively.

of high fertility areas under and around plant canopies (characterized by higher production of above- and belowground litter, leachates, and exudates and lower erosion rates), and low fertility areas in the zones devoid of perennial vascular vegetation (Schlesinger et al. 1990, Hook et al. 1991, Ochoa-Hueso et al. 2018). Our results provide partial empirical support for this hypothesis at a global scale in dryland ecosystems and confirm that any 
impact of climatic variation on vegetation spatial variability might also have significant consequences for SVM globally.

Our mechanistic model suggests that total plant cover has limited direct effects on SVM (Fig. 3). However, a higher number of plant patches in our plots was strongly and directly linked to lower levels of SVM. Thus, it seems that more even inputs of litter and a higher capacity to redistribute soil nutrients spatially, as a result of a more homogeneous distribution of plant patches, are more important than the total area covered by plants to maintain lower levels of soil spatial variability, at least at the scale evaluated in this study. Previous studies show that increases in aridity, or other disturbances such as overgrazing, are tightly linked to plant cover losses and to increases in the percentage of sand-sized particles in the soil (Schlesinger et al. 1990, Li et al. 2007). Here, we show that increases in temperature and aridity such as those forecasted with climate change, and the likely concomitant decreases in total plant cover and increases in the amount of sand in the soil might result in a general increase in the spatial variability of soil resources and functionality in drylands worldwide (Vicente-Serrano et al. 2012, DelgadoBaquerizo et al. 2013). Perennial plants modify soil texture by decreasing losses (or increasing the accumulation) of fine soil particles, thus decreasing the relative abundance of sand-sized grains in the soil (Linstädter and Baumann 2013). This process is likely to increase the functional differences between vegetated areas (where plants typically drive soil biological processes and promote soil nutrient redistribution via roots and microorganisms; Schlesinger et al. 1990, Hook et al. 1991), and open areas between plant patches dominated by physical processes such as wind and water erosion ( $\mathrm{Li}$ et al. 2007).

Soil heterogeneity drives many key ecosystem processes (Farley and Fitter 1999, Day et al. 2003, Maestre and Reynolds 2006, Zuo et al. 2010, Tsunoda et al. 2014), but predicting the effects of the observed changes in SVM is not trivial. According to the traditional view of spatial heterogeneity as a driver of species diversity, increased SVM should promote plant and soil biota diversity (Bakker et al. 2003, Davies et al. 2005). Recent studies suggest that, more likely, SVM can both increase and decrease not only diversity, but also ecosystem function, depending on factors such as the scale of the heterogeneity, the environmental conditions, as well as species identity and composition (Hutchings et al. 2003). Increases in SVM in low productivity systems such as drylands could change the size symmetry of belowground competition, favoring larger (in the case of plants) or more mobile (in the case of soil biota) species, which are more capable of rapidly exploiting nutrient patches than smaller species (Rajaniemi 2007, Reynolds and Haubensak 2009). On the other hand, changes in SVM typically promote multiple plant morphological and physiological responses, such as changes in nutrient uptake kinetics, biomass allocation, and root production and morphology (Robinson 1994, García-Palacios et al. 2012). Thus, the ability of individual species (and individuals within species) to adapt to the forecasted increase (with climate change) in soil spatial variability will likely determine their establishment (Maestre et al. 2003), competitive ability (Robinson et al. 1999, Hodge 2004), productivity (Dougill and Thomas 2004), and survival rate (Wijesinghe et al. 2005).

Of particular interest were the different responses of the element-specific N-, C-, and P-SVM to the environmental predictors. The predictive capacity of our model was clearly higher for N-SVM than for C- and P-SVM. While we also found strong direct effects of aridity on N-SVM, the fate of C- and P-SVM was mainly indirectly driven by reductions in number of plant patches. Different ecosystem compartments or processes may have different sensitivities to the direct or indirect effects of aridity (Evans and Burke 2012), and past studies have shown asymmetrical responses of $\mathrm{N}$, $\mathrm{C}$, and $\mathrm{P}$ cycles to climate change, with $\mathrm{N}$ cycling being consistently the most susceptible among them (Durán et al. 2013). Several mechanisms linked to increases in aridity and temperature could be behind this different sensitivity of the spatial variability of $\mathrm{N}, \mathrm{C}$, and $\mathrm{P}$ in soils. For instance, different microbial communities have different sensitivities to warming and drought, leading to the accumulation of different soil nutrient pools (Sheik et al. 2011). Also, unlike $\mathrm{P}$ and $\mathrm{C}$, whose availabilities are principally linked to the parent material and the decomposition of litter from plant communities, respectively, soil $\mathrm{N}$ is fixed from the atmosphere by soil microbial communities (e.g., most cyanobacteria), which are common in many dryland soils (Schlesinger and Bernhardt 2013). Increases in aridity will increase the amount of potential habitat available for biocrust communities, which spread in the open areas between plant patches and form mosaics of multiple species (Delgado-Baquerizo et al. 2016a). Similarly, increases in aridity promote the abundance of autotrophic communities tightly linked to the N cycle (e.g., archaeal nitrifiers; Delgado-Baquerizo et al. 2016b). Thus, the strong links between aridity and the spatial distribution of $\mathrm{N}$ cyclingrelated microbial communities might help explain the strong direct effect of climate on N-SVM, not observed for $\mathrm{P}$ - or C-SVM. On the contrary, the strong indirect effects of aridity on C- and P-SVM via reductions in number of plant patches might be related to reduced litter decomposition, as our C- and P-SVM indexes include the activities of enzymes, such as beta-glucosidase and phosphatase, that are involved in the degradation of organic matter into simpler $\mathrm{C}$ and $\mathrm{P}$ components.

The biogeochemical cycles of $\mathrm{N}, \mathrm{C}$, and $\mathrm{P}$ are tightly interlinked in terrestrial ecosystems by processes such as photosynthesis, atmospheric $\mathrm{N}$ fixation, respiration, decomposition, and microbial mineralization (Vitousek 2004, Schlesinger and Bernhardt 2013). However, as these processes are likely to be altered, perhaps in different ways, by anthropogenic disturbances such as climate change, it has been suggested that N, C, and P cycles can become decoupled (Schlesinger et al. 1990, Peñuelas et al. 2012, Vicente-Serrano et al. 2012, Delgado-Baquerizo et al. 2013). Here we show that increasing aridity and temperature had stronger effects on the heterogeneity of $\mathrm{N}$ - than $\mathrm{C}$ and P-related variables. These results suggest that expected changes in climate, by compromising the essential co-occurrence of areas with similar N, C, and P contents, might lead to a decoupling of the spatial availability of these elements for plants and microbes in dryland soils across the globe. 
Heterogeneity is rarely employed as a response variable to assess the effects of human impacts on ecosystems, but recent studies indicate that it can be a sensitive metric per se, capturing effects and differences sometimes not detected by averaging (Underwood 1991, Callaghan and Holloway 1999, Fraterrigo and Rusak 2008). Indeed, whereas a recent study using our same database showed that $\mathrm{C}$ and $\mathrm{N}$ cycles are likely to become uncoupled from the $\mathrm{P}$ cycle in coming decades due to increasing aridity (Delgado-Baquerizo et al. 2013), our explicit consideration of SVM unveiled an additional (spatial) C-N-P decoupling mechanism that could have important consequences for ecosystem functioning (Schimel 2010, Finzi et al. 2011, Peñuelas et al. 2012, Delgado-Baquerizo et al. 2013).

Together, our work provides the first empirical evidence that changes in temperature and aridity are linked to alterations of the SVM in drylands across the globe. Our results also confirm that the direction of these effects is maintained when analyzing the spatial variability of $\mathrm{N}, \mathrm{C}$, and $\mathrm{P}$ variables independently. However, the spatial variability of $\mathrm{N}$ cycling processes was more sensitive to changes in temperature and aridity than that of C-, and P-cycling. Whereas the effects of aridity and temperature on N-SVM were mainly direct, in the case of C-SVM and P-SVM these effects were indirectly driven by reductions in the number of plant patches promoted by aridity. These findings significantly advance our understanding of the patterns and mechanisms driving the spatial heterogeneity of soil multifunctionality across the globe, which is critical for understanding the responses of terrestrial ecosystems to ongoing climate change.

\section{ACKNOWLEDGMENTS}

This research was supported by the European Research Council (ERC) under the European Community's Seventh Framework Programme (FP7/2007-2013)/ERC grant agreement no. 242658 (BIO$\mathrm{COM})$, and by the Spanish Ministry of Economy and Competitiveness (BIOMOD, ref. CGL2013-44661-R). J. Durán acknowledges support from the Fundação para Ciência e Tecnologia (IF/00950/2014). M. Delgado-Baquerizo acknowledges support from the Marie Sklodowska-Curie Actions of the Horizon 2020 Framework Program H2020-MSCA-IF-2016 under REA grant agreement no 702057. A. Linstädter and R. T. Guuroh acknowledge support of the German Federal Ministry of Education and Research (BMBF) through WASCAL (grant 01LG1202-A). F. T. Maestre acknowledges support from the BIODESERT project (ERC grant agreement no. 647038).

\section{Literature Cited}

Allen, S. E., H. M. Grimshaw, and A. P. Rowland. 1986. Chemical analysis. Pages 285-344 in P. D. Moore, editor. Methods in plant ecology. Blackwell Scientific Publications, Oxford, UK.

Anderson, J. M., and J. S. Ingram. 1993. Tropical soil biology and fertility: a handbook of methods. Second edition. CABI Publishing, Wallingford, UK.

Bakker, C., J. M. Blair, and A. K. Knapp. 2003. Does resource availability, resource heterogeneity or species turnover mediate changes in plant species richness in grazed grasslands? Oecologia 137:385-391.

Breiman, L. 2001. Random forests. Machine Learning 45:5-32.

Brun, J. M., and T. W. Box. 2006. A comparison of line intercepts and random point frames for sampling desert shrub vegetation.
Rangeland Ecology \& Management/Journal of Range Management Archives 16:21-25.

Byrnes, J. E. K., L. Gamfeldt, F. Isbell, J. S. Lefcheck, J. N. Griffin, A. Hector, B. J. Cardinale, D. U. Hooper, L. E. Dee, and J. Emmett Duffy. 2014. Investigating the relationship between biodiversity and ecosystem multifunctionality: challenges and solutions. Methods in Ecology and Evolution 5:111-124.

Callaghan, A., and G. J. Holloway. 1999. The relationship between environmental stress and variance. Ecological Applications 9:456-462.

Davies, K. F., P. Chesson, S. Harrison, B. D. Inouye, B. A. Melbourne, and K. J. Rice. 2005. Spatial heterogeneity explains the scale dependence of the native-exotic diversity relationship. Ecology 86:1602-1610.

Day, K. J., M. J. Hutchings, and E. A. John. 2003. The effects of spatial pattern of nutrient supply on yield, structure and mortality in plant populations. Journal of Ecology 91:541-553.

Delgado-Baquerizo, M., et al. 2013. Decoupling of soil nutrient cycles as a function of aridity in global drylands. Nature 502:672676.

Delgado-Baquerizo, M., P. García-Palacios, R. Milla, A. Gallardo, and F. T. Maestre. 2015. Soil characteristics determine soil carbon and nitrogen availability during leaf litter decomposition regardless of litter quality. Soil Biology and Biochemistry 81:134 142.

Delgado-Baquerizo, M., F. T. Maestre, D. J. Eldridge, M. A. Bowker, V. Ochoa, B. Gozalo, M. Berdugo, J. Val, and B. K. Singh. 2016a. Biocrust-forming mosses mitigate the negative impacts of increasing aridity on ecosystem multifunctionality in drylands. New Phytologist 209:1540-1552.

Delgado-Baquerizo, M., F. T. Maestre, D. J. Eldridge, and B. K. Singh. 2016b. Microsite differentiation drives the abundance of soil ammonia oxidizing bacteria along aridity gradients. Frontiers in Microbiology 7:505.

Dougill, A. J., and A. D. Thomas. 2004. Kalahari sand soils: spatial heterogeneity, biological soil crusts and land degradation. Land Degradation \& Development 15:233-242.

Duarte, C. M. 1991. Variance and the description of nature. Pages 301-318 in J. Cole, G. M. Lovett and S. Findlay, editors. Comparative analyses of ecosystems. Springer-Verlag, New York, New York, USA.

Durán, J., A. Rodríguez, J. L. Morse, and P. M. Groffman. 2013. Winter climate change effects on soil $\mathrm{C}$ and $\mathrm{N}$ cycles in urban grasslands. Global Change Biology 19:2826-2837.

Evans, S. E., and I. C. Burke. 2012. Carbon and nitrogen decoupling under an 11-year drought in the shortgrass steppe. Ecosystems $16: 20-33$.

Farley, R. A., and A. H. Fitter. 1999. Temporal and spatial variation in soil resources in a deciduous woodland. Journal of Ecology 87:688-696.

Finzi, A. C., A. T. Austin, E. E. Cleland, S. D. Frey, B. Z. Houlton, and M. D. Wallenstein. 2011. Responses and feedbacks of coupled biogeochemical cycles to climate change: examples from terrestrial ecosystems. Frontiers in Ecology and the Environment 9:61-67.

Fraterrigo, J. M., and J. A. Rusak. 2008. Disturbance-driven changes in the variability of ecological patterns and processes. Ecology Letters 11:756-770.

Gao, X., and F. Giorgi. 2008. Increased aridity in the Mediterranean region under greenhouse gas forcing estimated from high resolution simulations with a regional climate model. Global and Planetary Change 62:195-209.

García-Palacios, P., F. T. Maestre, R. D. Bardgett, and H. de Kroon. 2012. Plant responses to soil heterogeneity and global environmental change. Journal of Ecology 100:1303-1314.

Grace, J. B. 2006. Structural equation modeling and natural systems. Cambridge University Press, Cambridge, UK.

Guuroh, R. T., J. C. Ruppert, J. Ferner, K. Čanak, S. Schmidtlein, and A. Linstädter. 2018. Drivers of forage provision and erosion 
control in West African savannas-A macroecological perspective. Agriculture, Ecosystems \& Environment 251:257-267.

Hijmans, R. J., S. E. Cameron, J. L. Parra, P. G. Jones, and A. Jarvis. 2005. Very high resolution interpolated climate surfaces for global land areas. International Journal of Climatology 25:19651978.

Hodge, A. 2004. The plastic plant: root responses to heterogeneous supplies of nutrients. New Phytologist 162:9-24.

Hook, P. B., I. C. Burke, and W. K. Lauenroth. 1991. Heterogeneity of soil and plant $\mathrm{N}$ and $\mathrm{C}$ associated with individual plants and openings in North American shortgrass steppe. Plant and Soil 138:247-256.

Huang, J., H. Yu, X. Guan, G. Wang, and R. Guo. 2016. Accelerated dryland expansion under climate change. Nature Climate Change 6:166-171.

Hutchings, M. J., E. A. John, and D. K. Wijesinghe. 2003. Toward understanding the consequences of soil heterogeneity for plant populations and communities. Ecology 84:23222334.

IPCC. 2013. Summary for Policymakers. Pages 1-30 in T. F. Stocker, D. Qin, G.-K. Plattner, M. Tignor, S. K. Allen, J. Boschung, A Nauels, Y. Xia, V. Bex, and P. M. Midgley, editors. Climate Change 2013: The Physical Science Basis. Contribution of Working Group I to the Fifth Assessment Report of the Intergovernmental Panel on Climate Change. Cambridge University Press, Cambridge, UK

Jackson, R. B., and M. M. Caldwell. 1993. The scale of nutrient heterogeneity around individual plants and its quantification with geostatistics. Ecology 74:612-614.

Jones, D. L., and V. B. Willett. 2006. Experimental evaluation of methods to quantify dissolved organic nitrogen (DON) and dissolved organic carbon (DOC) in soil. Soil Biology and Biochemistry 38:991-999.

Kettler, T. A., J. W. Doran, and T. L. Gilbert. 2001. Simplified method for soil particle-size determination to accompany soilquality analyses. Soil Science Society of America Journal 65:849852.

Li, J., G. S. Okin, L. Alvarez, and H. Epstein. 2007. Quantitative effects of vegetation cover on wind erosion and soil nutrient loss in a desert grassland of southern New Mexico, USA. Biogeochemistry 85:317-332.

Linstädter, A., and G. Baumann. 2013. Abiotic and biotic recovery pathways of arid rangelands: lessons from the High Atlas Mountains, Morocco. Catena 103:3-15.

Linstädter, A., J. Schellberg, K. Brüser, C. A. M. García, R. J. Oomen, C. C. du Preez, J. C. Ruppert, and F. Ewert. 2014. Are there consistent grazing indicators in drylands? Testing plant functional types of various complexity in South Africa's grassland and Savanna biomes. PLoS ONE 9:e104672.

Maestre, F. T., and J. F. Reynolds. 2006. Biomass responses to elevated $\mathrm{CO} 2$, soil heterogeneity and diversity: an experimental assessment with grassland assemblages. Oecologia 151:512 520

Maestre, F. T., J. Cortina, S. Bautista, J. Bellot, and R. Vallejo. 2003. Small-scale environmental heterogeneity and spatiotemporal dynamics of seedling establishment in a semiarid degraded ecosystem. Ecosystems 6:630-643.

Maestre, F. T., et al. 2012. Plant species richness and ecosystem multifunctionality in global drylands. Science 335:214 218.

Moir, J., and H. Tiessen. 2007. Characterization of available P by sequential extraction. Page 293-306 in M. R. Carter, and E. G. Gregorich, editors. Soil sampling and methods of analysis. Second Edition. CRC Press, Boca Raton, Florida, USA.

Moles, A. T., D. I. Warton, L. Warman, N. G. Swenson, S. W. Laffan, A. E. Zanne, A. Pitman, F. A. Hemmings, and M. R. Leishman. 2009. Global patterns in plant height. Journal of Ecology 97:923-932.
Ochoa-Hueso, R., et al. 2018. Soil fungal abundance and plant functional traits drive fertile island formation in global drylands. Journal of Ecology 106:242-253.

Peñuelas, J., J. Sardans, A. Rivas-ubach, and I. A. Janssens. 2012. The human-induced imbalance between C, N and P in Earth's life system. Global Change Biology 18:3-6.

Prăvălie, R. 2016. Drylands extent and environmental issues. A global approach. Earth-Science Reviews 161:259-278.

Rajaniemi, T. K. 2007. Root foraging traits and competitive ability in heterogeneous soils. Oecologia 153:145-152.

Reynolds, H. L., and K. A. Haubensak. 2009. Soil fertility, heterogeneity, and microbes: towards an integrated understanding of grassland structure and dynamics. Applied Vegetation Science 12:33-44.

Robinson, D. 1994. The responses of plants to non-uniform supplies of nutrients. New Phytologist 127:635-674.

Robinson, D., A. Hodge, B. S. Griffiths, and A. H. Fitter. 1999. Plant root proliferation in nitrogen-rich patches confers competitive advantage. Proceedings of the Royal Society B 266:431.

Schermelleh-Engel, K., H. Moosbrugger, and H. Müller. 2003. Evaluating the fit of structural equation models: tests of significance and descriptive goodness-of-fit measures. Methods of Psychological Research-Online 8:23-74.

Schimel, D. S. 2010. Drylands in the Earth System. Science 327:418-419.

Schlesinger, W. H., and E. S. Bernhardt. 2013. Biogeochemistry an analysis of global change. Academic Press, Cambridge, Massachusetts, USA.

Schlesinger, W. H., J. F. Reynolds, G. L. Cunningham, L. F. Huenneke, W. M. Jarrell, R. A. Virginia, and W. G. Whitford. 1990. Biological feedbacks in global desertification. Science 247:10431048

Sheik, C. S., W. H. Beasley, M. S. Elshahed, X. Zhou, Y. Luo, and L. R. Krumholz. 2011. Effect of warming and drought on grassland microbial communities. ISME Journal 5:1692-1700.

Tabatabai, M. A. 1982. Methods in soil analyses. Part 2. American Society of Agronomy, Madison, Wisconsin, USA.

Tabatabai, M. A., and J. M. Bremner. 1969. Use of p-nitrophenyl phosphate for assay of soil phosphatase activity. Soil Biology and Biochemistry 1:301-307.

Tsunoda, T., N. Kachi, and J.-I. Suzuki. 2014. Interactive effects of soil nutrient heterogeneity and belowground herbivory on the growth of plants with different root foraging traits. Plant and Soil 384:327-334

Underwood, A. J. 1991. Beyond BACI: Experimental designs for detecting human environmental impacts on temporal variations in natural populations. Australian Journal of Marine and Freshwater Research 42:569-587.

Vicente-Serrano, S. M., A. Zouber, T. Lasanta, and Y. Pueyo. 2012. Dryness is accelerating degradation of vulnerable shrublands in semiarid Mediterranean environments. Ecological Monographs 82:407-428.

Vitousek, P. M. 2004. Nutrient cycling and limitation: Hawai'i as a model system. Princeton University Press, Princeton, New Jersey, USA.

Wijesinghe, D. K., E. A. John, and M. J. Hutchings. 2005. Does pattern of soil resource heterogeneity determine plant community structure? An experimental investigation. Journal of Ecology 93:99-112.

Zomer, R. J., A. Trabucco, L. V. Verchot, and B. Muys. 2008. Land area eligible for afforestation and reforestation within the clean development mechanism: a global analysis of the impact of forest definition. Mitigation and Adaptation Strategies for Global Change 13:219-239.

Zornoza, R., C. Guerrero, J. Mataix-Solera, V. Arcenegui, F. García-Orenes, and J. Mataix-Beneyto. 2006. Assessing air-drying and rewetting pre-treatment effect on some soil enzyme activities 
under Mediterranean conditions. Soil Biology and Biochemistry 38:2125-2134.

Zornoza, R., J. Mataix-Solera, C. Guerrero, V. Arcenegui, and J. Mataix-Beneyto. 2009. Storage effects on biochemical properties of air-dried soil samples from Southeastern Spain. Arid Land Research and Management 23:213-222.
Zuo, X. A., X. Y. Zhao, H. L. Zhao, Y. R. Guo, T. H. Zhang, and J. Y. Cui. 2010. Spatial pattern and heterogeneity of soil organic carbon and nitrogen in sand dunes related to vegetation change and geomorphic position in Horqin Sandy Land, Northern China. Environmental Monitoring and Assessment 164:29-42.

\section{SUPPORTING INFORMATION}

Additional supporting information may be found in the online version of this article at http://onlinelibrary.wiley.com/doi/10.1002/ecy. 2199/suppinfo

\section{Data Availability}

Data associated with this study are available in Figshare: https://doi.org/10.6084/m9.figshare.5789079. 